\title{
Bilateral Serous Retinal Detachment in Lymphoid Blast Crisis of Chronic Myeloid Leukemia
}

\author{
Kyohei Umemura $^{a} \quad K^{\prime} y o k o$ Fujita ${ }^{a} \quad$ Akiko Jinno $^{a}$ Ayano Nakamura ${ }^{b}$ \\ Motohiro Kamei ${ }^{a}$ \\ aDepartment of Ophthalmology, Aichi Medical University, Nagakuteshi, Japan; \\ ${ }^{b}$ Department of Hematology, Aichi Medical University, Nagakuteshi, Japan
}

\section{Keywords}

Serous retinal detachment $\cdot$ Leukemia $\cdot$ Blast crisis

\begin{abstract}
We herein report a patient with Philadelphia chromosome-positive lymphoid blast crisis of chronic myeloid leukemia $(\mathrm{CML})$, who presented with bilateral serous retinal detachment (SRD). A 36-year-old Asian male presented with the symptoms of decreased vision and was found to have bilateral SRD involving fovea. There was no inflammation in the anterior chamber or vitreous. Physical examination showed hepatomegaly and splenomegaly. A blood count revealed white blood cell count of $38.2 \times 10^{9} / \mathrm{L}$ with $51.5 \%$ blast cells. Bone marrow aspirate showed total cell count of $145 \times 10^{3} / \mu \mathrm{L}$ with $80.6 \%$ blast cells and negative neutrophil myeloperoxidase staining. Cytogenetic analysis using fluorescence in situ hybridization confirmed a 9;22 chromosomal translocation, indicating the presence of the Philadelphia chromosome. Flow cytometry analysis demonstrated expression of CD10, CD19, and positive TdT. According to morphology, immunology, cytogenetics, and molecular criteria, the patient was diagnosed as having Philadelphia chromosome-positive lymphoid blast crisis of CML. Based on the ocular findings and hematological abnormalities, the SRD was considered to be ocular involvement secondary to the blast crisis of leukemia. Two months after starting induction therapy, fundus examination and optical coherence tomography showed complete resolution of bilateral SRD and improved vision. Prompt diagnosis of the disease leads to early systemic chemotherapy and may help restore visual function and improve survival.
\end{abstract}




\section{Introduction}

Ophthalmic manifestations of leukemia are frequently encountered in the clinical setting. Although ocular manifestations may occur in all types of leukemia, ocular involvement in lymphoid blast crisis of chronic myeloid leukemia (CML) is rarely reported. We herein report a patient with Philadelphia chromosome-positive lymphoid blast crisis of CML, who presented with bilateral serous retinal detachment (SRD).

\section{Case Report}

A 36-year-old Asian male was admitted to our hospital with severe general fatigue. Two months before presentation, he was admitted to a local clinic with fever and was diagnosed with anemia, and received blood transfusions. Despite this treatment course, his symptoms worsened, and he consulted our hospital. Physical examination showed hepatomegaly and splenomegaly. Complete blood count revealed red blood cell count of $1.81 \times 10^{12} / \mathrm{L}$ and hemoglobin level of $54 \mathrm{~g} / \mathrm{L}$, confirming anemia; platelet count of $214 \times 10^{9} / \mathrm{L}$ and severe leukocytosis with white blood cell count of $38.2 \times 10^{9} / \mathrm{L}$. Differential count was $15 \%$ neutrophils, $24.5 \%$ lymphocytes, 4.5\% monocytes, $1.0 \%$ eosinophils, $0 \%$ basophils, $1 \%$ promyelocytes, $2 \%$ myelocytes, and $51.5 \%$ blast cells. Bone marrow aspirate showed total cell count of $145 \times 10^{3} / \mu \mathrm{L}$ with $80.6 \%$ blast cells, and negative neutrophil myeloperoxidase staining. Cytogenetic analysis using fluorescence in situ hybridization confirmed a 9;22 chromosomal translocation, indicating the presence of the Philadelphia chromosome. Flow cytometry analysis demonstrated expression of CD10, CD19, and positive TdT. Examination of cerebrospinal fluid was negative for malignant cells. According to morphology, immunology, cytogenetics, and molecular criteria, the patient was diagnosed as having a blast crisis of Philadelphia-positive acute lymphoblastic leukemia.

Additionally, he also complained of decreased vision 1 month before presentation at our hospital. He was seen at the ophthalmology outpatient clinic of our hospital one day after starting oral corticosteroids as treatment for leukemia. On ocular examination, decimal bestcorrected visual acuity was 1.2 in the right eye (OD) and 0.8 in the left eye (OS), and intraocular pressure was within normal limits bilaterally (OU). Slit-lamp examination revealed no anterior chamber cells and flare, and no vitreous cells. Fundus examination revealed bilateral SRD involving the fovea and some retinal hemorrhages and Roth spots in peripheral retina OU (Fig. 1). Optical coherence tomography (OCT) showed subretinal fluid accumulation and thickened choroids OU. Choroidal thickness could not be measured because the choroid-scleral interface was not visible due to shadowing OU. No subretinal septa were observed OU. Fluorescein angiography (FA) showed multiple, pinpoint hyperfluorescent spots in the early phase, pooling of the fluorescein dye in subretinal space, and leakage from the optic disc in the late phase. Differential diagnosis included Vogt-Koyanagi-Harada (VKH) disease and central serous chorioretinopathy. Although the patient's FA findings were almost the same as those of VKH disease, he showed no inflammatory sign, prodromal meningeal irritation, and inner ear disturbance, and his OCT images did not show subretinal septa. Based on the ocular findings and hematological abnormalities, the SRD was considered to be ocular involvement secondary to the blast crisis of leukemia.

After receiving oral prednisolone (starting $95 \mathrm{mg} /$ day) for 7 days as prior induction chemotherapy, the patient started induction therapy with the standard protocol, consisting of imatinib, daunorubicin, vincristine, and cyclophosphamide. Two months after starting induction therapy, fundus examination and OCT showed complete resolution of bilateral SRD; the choroid-scleral interface was visible, and the choroidal thickness at the fovea was $458 \mu \mathrm{m}$ OD and $448 \mu \mathrm{m}$ OS (Fig. 2). Best-corrected visual acuity improved to $1.2 \mathrm{OD}$ and 1.0 OS. Eight

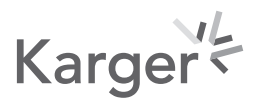



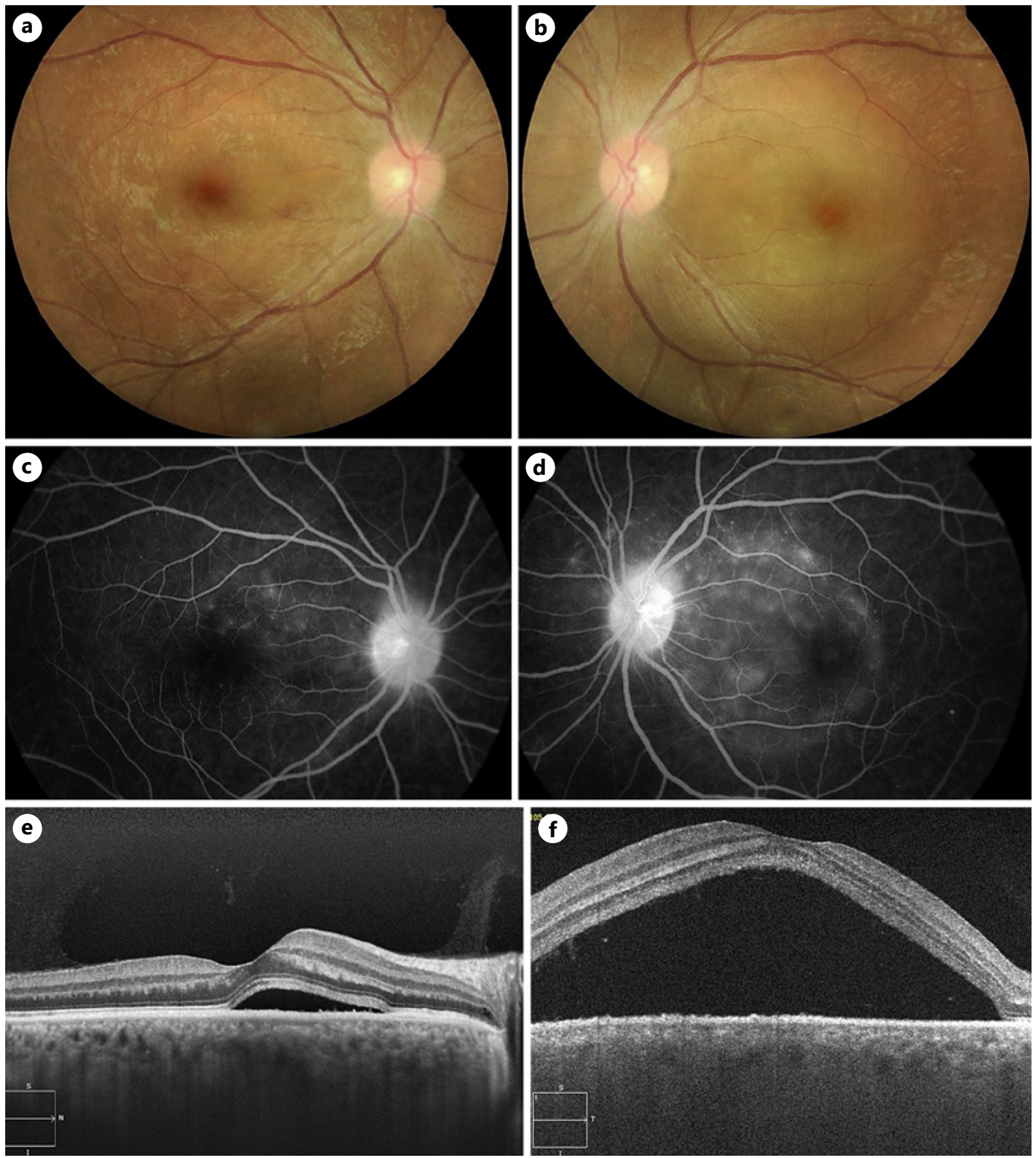

Fig. 1. Ocular findings at presentation. Fundus photographs show SRD at the posterior pole in the OD (a) and OS (b). c, d FA in middle phase shows multiple hyperfluorescent pinpoint leakages and leakage from the optic disc in both eyes. OCT shows subretinal fluid in the OD (e) and OS (f). SRD, serous retinal detachment; OD, right eye; OS left eye; OCT, optical coherence tomography; FA, fluorescein angiography.

months after the first course of chemotherapy, SRD remained resolved. No sunset glow appearance of the fundus was observed OU (Fig. 3).

\section{Discussion/Conclusion}

Ophthalmic involvement of leukemia can occur either primarily by direct infiltration of leukemic cells in ocular tissues or secondarily due to hematologic abnormalities, central nerve system (CNS) involvement, and toxicity of various chemotherapeutic drugs [1]. In the present 

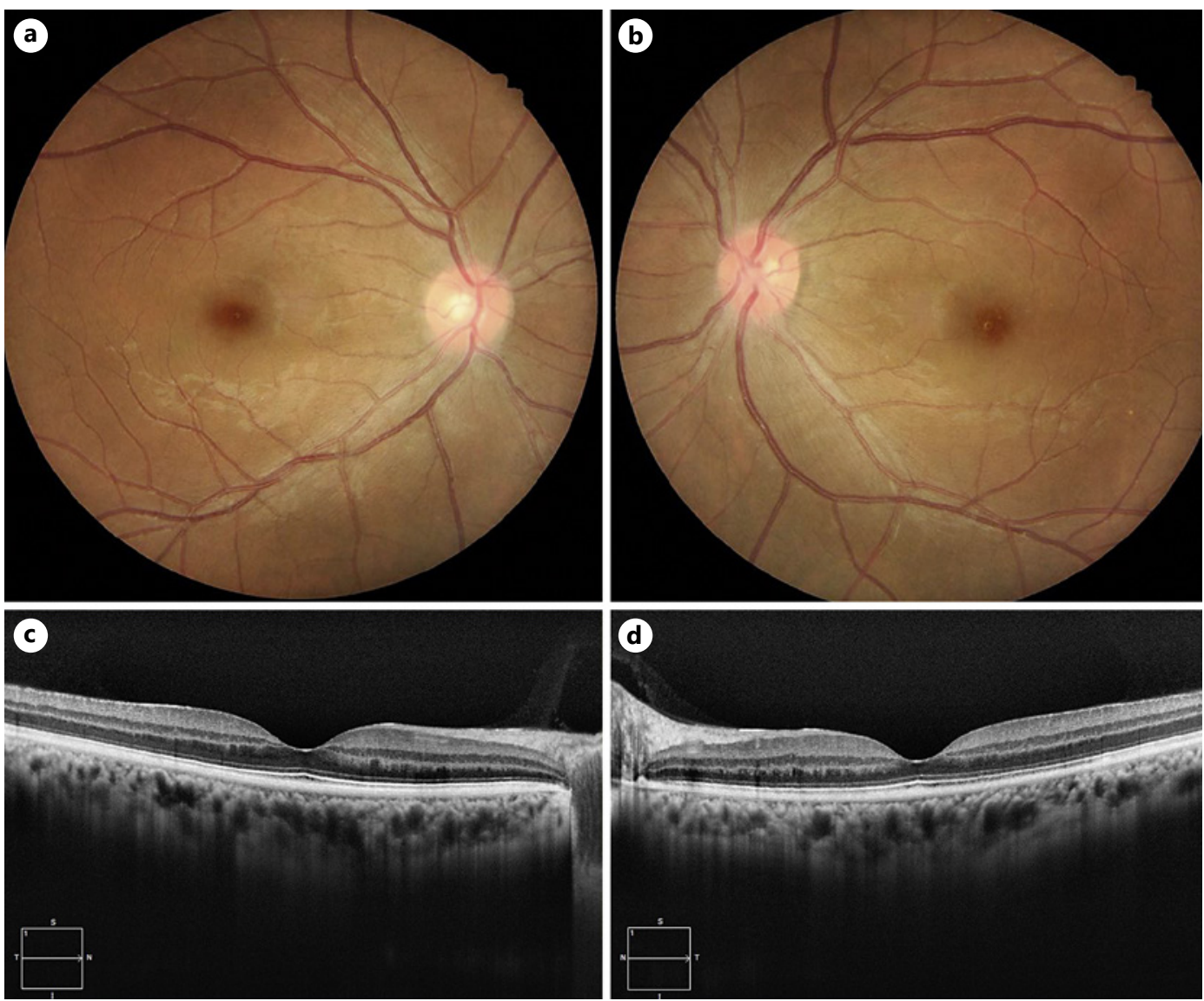

Fig. 2. Ocular findings at 2 months after start of induction chemotherapy. Fundus photographs show complete resolution of SRD in the OD (a) and OS (b). OCT shows complete resolution of subretinal fluid in the OD (c) and OS (d). SRD, serous retinal detachment; OD, right eye; OS, left eye; OCT, optical coherence tomography.

case, SRD occurred as an extramedullary manifestation. Although SRD may occur in any type of leukemia, ocular manifestations occurring during lymphoid blast crisis of CML are rarely reported [2-6]. Previous reports of ocular involvements in blast crisis of CML included masquerade syndrome [2]; infiltration of leukemia cells to the iris and choroid [3]; infiltration of vitreous [4]; and choroidal infiltration and panuveitis with leukemic cells in either anterior chamber or the vitreous, retinal hemorrhages, and SRD [5]. Reports of SRD alone as ocular manifestation are even rarer [6]. The reason for the variety of ocular manifestations in blast crisis of CML is unclear.

Several previous reports have also described SRD in leukemia [7-12]. The hypotheses for the pathogenesis of SRD in leukemia include sequestration of infiltrated leukemic cells in the choroid and occlusion of the choriocapillaris. As a result of the latter, the interference with blood supply to the retinal pigment epithelium may cause secondary retinal pigment epithelium dysfunction [7-12].

Leukemia presenting as SRD needs to be differentiated from other etiologies that present as SRD, such as VKH disease [11,12]. Our case did not show inflammatory signs in the anterior chamber or vitreous; OCT findings showed no subretinal septa generally observed in VKH disease; and there were no prodromal symptoms. Therefore, VKH disease was less probable.

In our case, leakage from optic nerve head was observed on FA. We speculate that involvement of the optic nerve head may be caused by direct optic nerve infiltration or increased intracranial pressure $[13,14]$. In the present case, no blast cells were detected in cytological analysis of the cerebrospinal fluid. However, there is still a possibility that blast 

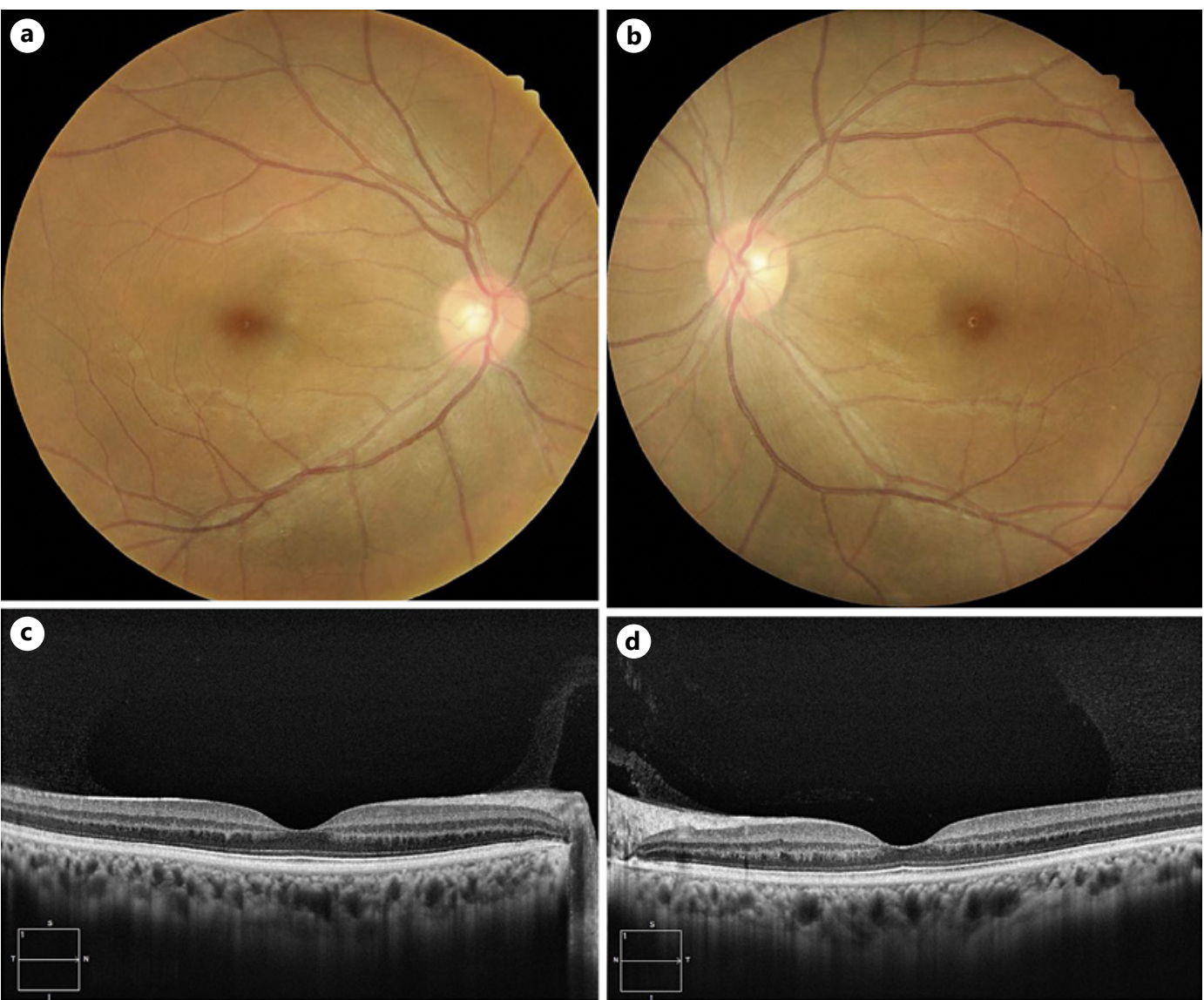

Fig. 3. Ocular findings at 8 months after start of induction chemotherapy. Fundus photographs show no abnormal findings in the OD (a) and OS (b). OCT shows no subretinal fluid in the OD (c) and OS (d). OD, right eye; OS, left eye; OCT, optical coherence tomography.

cells could have infiltrated the optic nerve because cerebrospinal fluid was examined after starting chemotherapy. The optic nerve is one of the sites of CNS leukemia presentation [15]; therefore, ophthalmologists should keep in mind to examine this structure and consider infiltrative causes as differential diagnosis for disc leakage. In conclusion, involvement of leukemia may be the first sign of extramedullary spread in the posterior segment of the eye and prompt diagnosis of the disease leads to early systemic chemotherapy and may help restore visual function and improve survival.

\section{Acknowledgement}

We would like to thank Prof. Akiyoshi Takami and Dr. Osami Daimaru for comments and advice. We also thank Dr. Teresa Nakatani for the English language review.

\section{Statement of Ethics}

The Ethics Committee of Aichi Medical University Hospital waived the need for approval of this study that involved a retrospective review of medical records. This report adhered to the tenets of the Declaration of Helsinki 1964. Because of the difficulty to obtain written 
informed consent from the patient due to his conditions, written informed consent was obtained from his wife for publication of this case report and any accompanying images.

\section{Conflict of Interest Statement}

The following authors have no financial disclosures: K.U., K.F., A.J., and A.N. M.K.: grants and personal fees from HOYA, grants and personal fees from Novartis Pharma KK, grants and personal fees from Pfizer Japan Inc., grants and personal fees from Santen, grants and personal fees from Senju, grants and personal fees from Alcon Japan, grants and personal fees from Bayer, grants and personal fees from Wakamoto Co. Ltd., grant fees from Abbott Medical Optics, grant fees from HANDAYA Co. Ltd., grant fees from TOMEY Co. Ltd., grant fees from Kawa Co. Ltd., grant fees from Otsuka Co. Ltd., grant fees from AMO Japan, grant fees from Alcon Pharma, grant fees from Chuo Sangyo Boueki, personal fees from Astellas Parma, personal fees from Nikon Health Care, personal fees from Leica microsystem, personal fees from Johnson \& Johnson, and personal fees from Abb Vie Inc., outside the submitted work.

\section{Funding Sources}

There was no funding for this article.

\section{Author Contributions}

K.U., K.F., A.J., and F.N. cared the patient, worked up, treated, and collected data. K.U., K.F., A.J., and M.K. analyzed the ophthalmological findings and gave critical suggestions. All the authors agree to be accountable for all aspects of work. All the authors approve the final version of the manuscript for publication.

\section{References}

1 Rosenthal AR. Ocular manifestations of leukemia. A review. Ophthalmology. 1983;90:899-905.

2 Lipton JH, McGowan HD, Payne DG. Ocular masquerade syndrome in lymphoid blast crisis of chronic myeloid leukemia. Leuk Lymphoma. 1995;20(1-2):161-3.

3 Gulati R, Alkhatib Y, Donthireddy V, Felicella MM, Menon MP, Inamdar KV. Isolated ocular manifestation of relapsed chronic myelogenous leukemia presenting as myeloid blast crisis in a patient on imatinib therapy: a case report and review of the literature. Case Rep Pathol. 2015;201524:380451.

4 Pleyer U, Weidle EG, Richter U, Haen M, Horny HP, Thiel HJ. Isolated infiltration of the vitreous body as a sign of blast crisis in chronic myeloid leukemia. Fortschr Ophthalmol. 1990;87:554-6.[German]

5 Lakosha H, Simpson R, Patterson B, Minden M, Payne D, Lipton JH. Acute lymphocytic blast crisis as an ocular manifestation of chronic granulocytic leukemia. Can J Ophthalmol. 2000;35:336-9.

6 Ohkoshi K, Yamaguchi T, Matsuba H, Kusano Y, Kanki K. Blastic crisis and bilateral serous retinal detachment in chronic myeloid leukemia. Rinsho Ganka. 1992;46:488-9.[Japanese]

7 Bajenova NV, Vanderbeek BL, Johnson MW. Change in choroidal thickness after chemotherapy in leukemic choroidopathy. Retina. 2012;32:203-5.

8 Miyamoto K, Kashii S, Honda Y. Serous retinal detachment caused by leukemic choroidal infiltration during complete remission. Br J Ophthalmol. 2000;84:1318-9.

9 Stewart MW, Gitter KA, Cohen G. Acute leukemia presenting as a unilateral exudative retinal detachment. Retina. 1989;9:110-4.

10 Green W, Rao PK, Harocopos GJ. Extramedullary relapse of acute myelogenous leukemia presenting as a large serous retinal detachment. Ocul Oncol Pathol. 2017;3(2):95-100.

11 Yang HK, Yu HG. Acute lymphoblastic leukemia manifesting as acute Vogt-Koyanagi-Harada disease. Korean J Ophthalmol. 2009;23:325-8. 
12 Kim J, Chang W, Sagong M. Bilateral serous retinal detachment as a presenting sign of acute lymphoblastic leukemia. Korean J Ophthalmol. 2010;24:245-8.

13 Yabas Kiziloglu O, Mestanoglu M, Totuk Gedar OM, Adiguzel C, Toygar O. Recurrence of acute lymphoblastic leukemia manifesting as serous retinal detachments and optic disc swelling. Int Ophthalmol. 2018;38:1791-5.

14 Brown GC, Shields JA, Augsburger JJ, Serota FT, Koch P. Leukemic optic neuropathy. Int Ophthalmol. 1981;3: 111-6.

15 Leonardy NJ, Rupani M, Dent G, Klintworth GK. Analysis of 135 autopsy eyes for ocular involvement in leukemia. Am J Ophthalmol. 1990;109:436-44. 